\title{
The role of cryptocurrencies in financial transactions considering modern global conditions
}

\author{
Fouad Eman M.* \\ Department of Economics, Faculty of Commerce (Girls), Al-Azhar University (Assiut Branch), 71524 Assiut, Egypt
}

\begin{abstract}
Despite all the fears surrounding cryptocurrencies, the rates of transactions increased and became an undeniable reality, and with the developments of the events that the world is going through, especially since the emergence of the new Corona virus and its declaration of a global pandemic, several important questions have emerged regarding dealing in these currencies considering the current epidemic. This study aims to know the extent to which dealing in cryptocurrencies has been affected by the emergence of the new Corona virus, and will it become a haven for transactions considering the closure decisions adopted by the countries of the world, or whether the uncertainty of the current situation increases the fear of dealing with it, especially as it depends on the forces of demand and supply. The study found that transactions in these currencies decreased at the beginning of the crisis, due to fears of a depreciation in their value, which prompted individuals to sell them on stock exchanges, but with the policies of closure and travel ban, dealing in these currencies increased again. Central banks tended strongly considering this crisis to think about maximizing benefit from dealing in these currencies.
\end{abstract}

Keywords: cryptocurrencies, blockchain, coronavirus, new monetary system.

* Corresponding author: Fouad Eman M.,

E-mail address: eman.mostafa@azhar.edu.eg 


\title{
دور العملات المشفرة فى التعاملات المالية فى ظل مستجدات الاوضاع العالمية
}

\author{
إيمان مصطفى فؤ اد \\ قسم الإقتصاد ، كلية التجارة (بنات) ، جامعة الأزهر (فرع أسيوط) ، أسيوط ، جمهورية مصر العربية
}

المستخلص

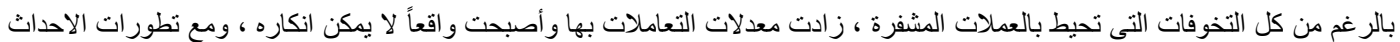

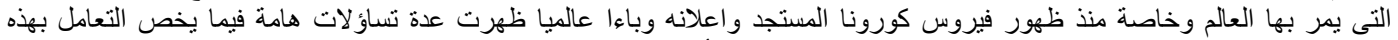

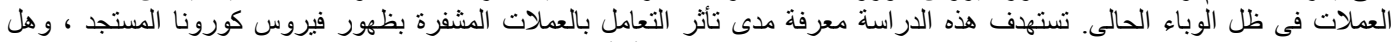

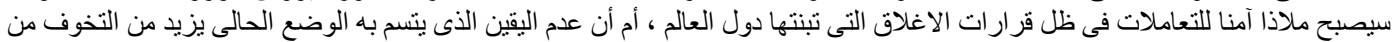

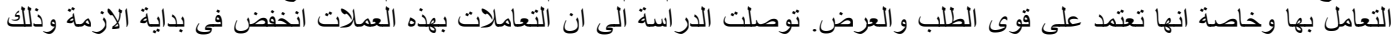

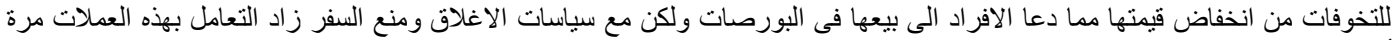

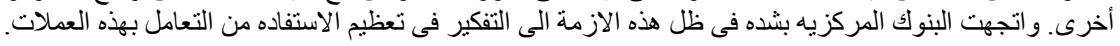




\section{مثكلة البحث}

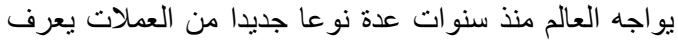

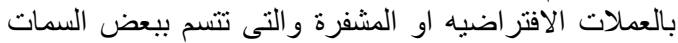

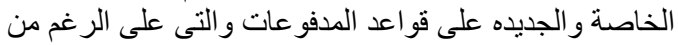

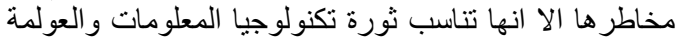

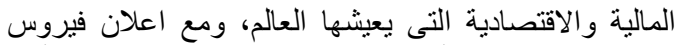

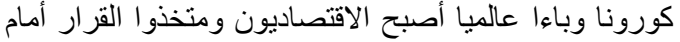

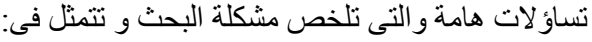
م هل تتأثر تعاملات العملات المشـفرة بتطور ات الاحداث

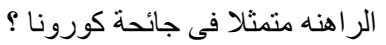

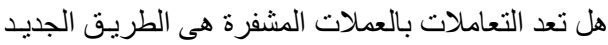

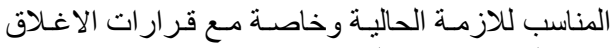
ومنع السفر وانتشار الوفيات ؟ لوالب

\section{خطة البحث}

تنقسم خطة البحث الى بيان ماهية العملات المشفرة

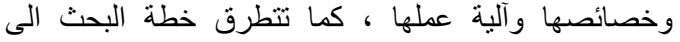

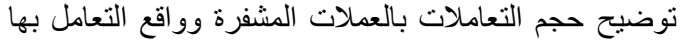

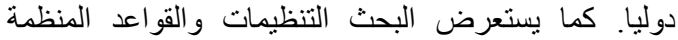

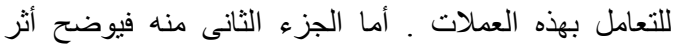
فيروس كورونا المستجد على التعاملات بالعملات المشفرة. لألهات

\section{النتائج ومناقشتها}

\section{أولا: ماهية العملات المشفرة وأشهرها}

أدت ثورة تكنولوجيا المعلومات الى ظهور ما عرف بالعملات

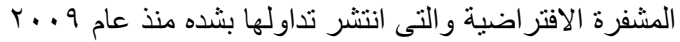

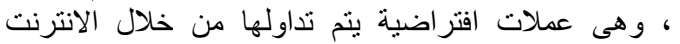

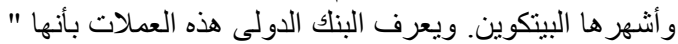

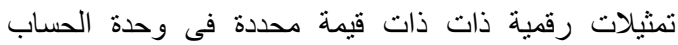

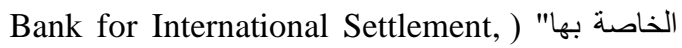

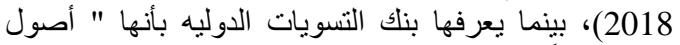

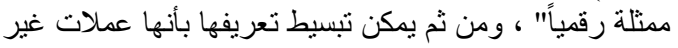
ملموسة تستخدم كوسيط للتبادل وتعتمد على على علم التشفير

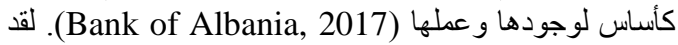
أصبحت العملات المشفرة معروضة للتداول فى سوق فوركس إنس

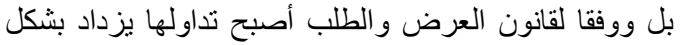

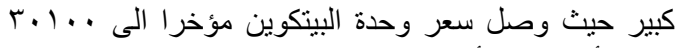

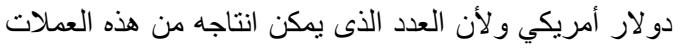

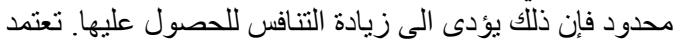

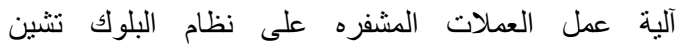

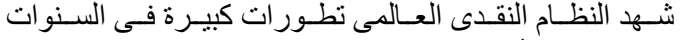

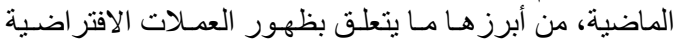

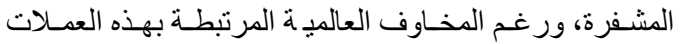

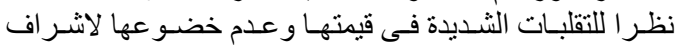

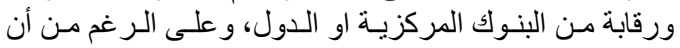

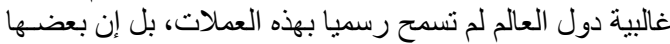

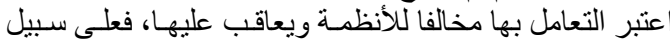

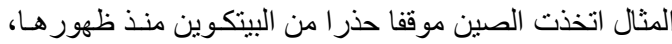

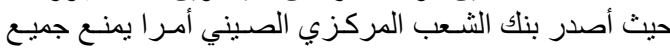

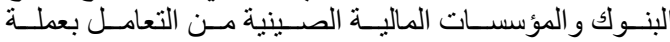

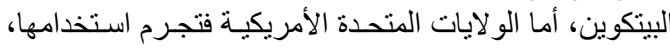

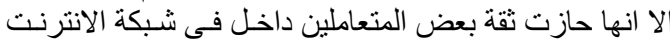

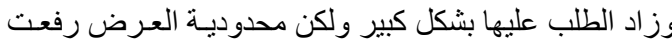

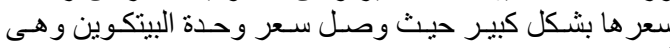

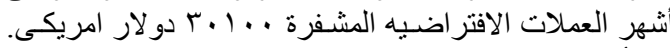

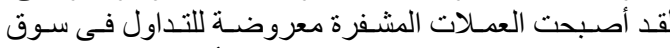

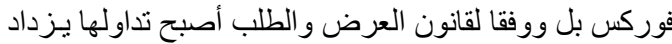

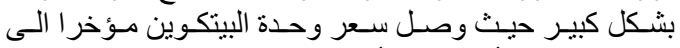

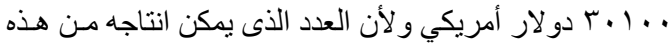

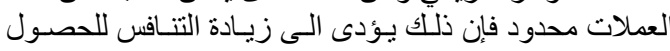

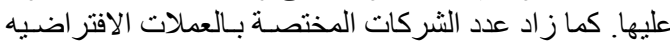

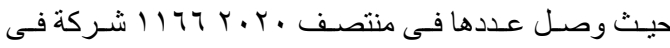

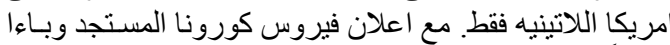

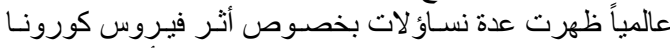

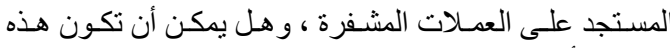

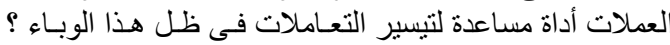

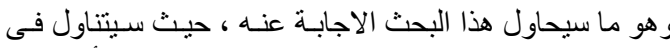

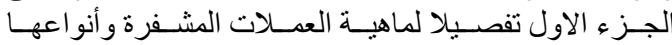

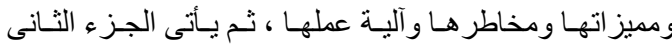
ليتناول وضع هذه العملات فى ظل انتشار هذا الفيروس.

هدف البحث

يهذف البحث الى استتعر اض مـدى تـأثير مستجدات الاوضـاع

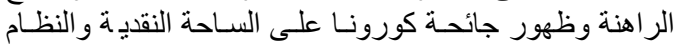

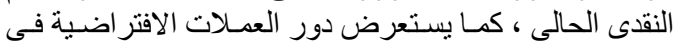

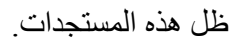

\section{أهمية البحث}

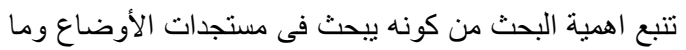

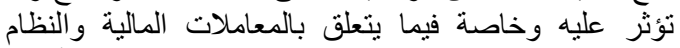

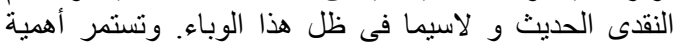

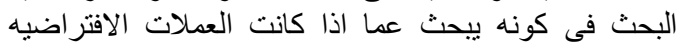

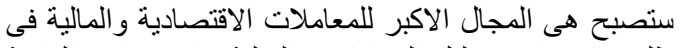
ظل وباء ينادى بتقليل المعامالات المالية والاقتصاديه المادية 
الطريقة من البنك المركزي إصدار كمية ثابتة من العملة

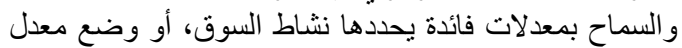

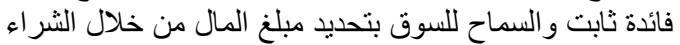

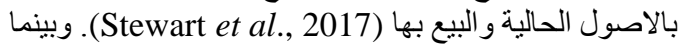

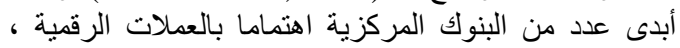

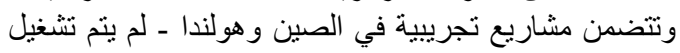

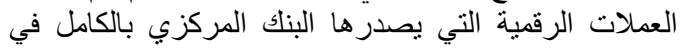
مجمو عة العشرين حتى الان.

\section{أ. آلية عمل العملات المشفرة}

تقوم آلية العمل أساسا على برنامج يتم تثبيته على جهاز

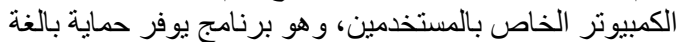

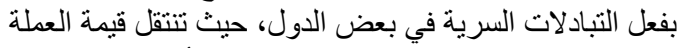

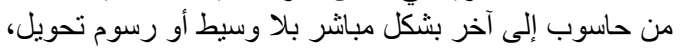
فبمجرد أن يقوم المستخدم بتحميل وتفعيل تطبيق البيتكوين يبدأ

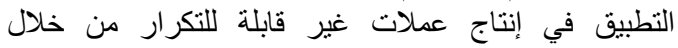
مبرمجات متخصصة يطلق عليها عمليات التعدين، ويتم

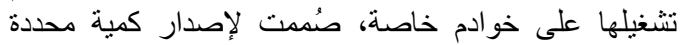

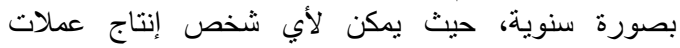

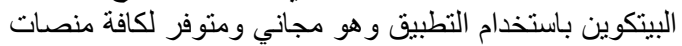

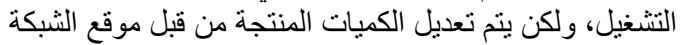

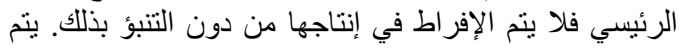
تخزين العملات التي تم إنتاجها في المحفظة الإلكترونية

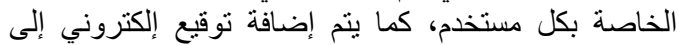

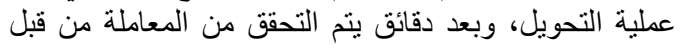

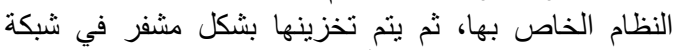
البيتكوين. ومن المفترض أن كل معاملة تتم بالبيتكوين يتم تسجيلها على البلوك تشين، حيث يتم تسجيل المبن المعاملات وتدوينها، و الحسابات التي يتم استخدامها في عملية التعدين، و التبادل، و عدد وحدات البيتكوين التي تم تداولها، وذلئ ولك لتحليل المعاملات و التأكد من عدم تكر اره.

\section{ب. مزايا التعامل بالعملات المشفرة}

تحمـل العمـــلات المشفرة (الافتر اضيــة) عـديد من المزايا

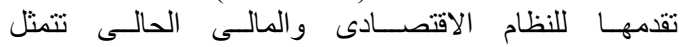
فى (Krause, 2016):

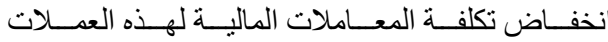

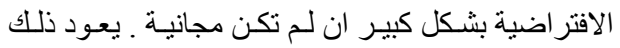

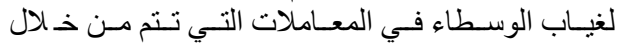

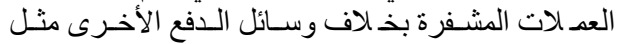

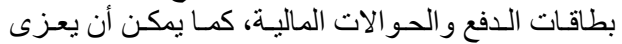

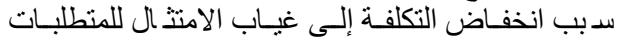

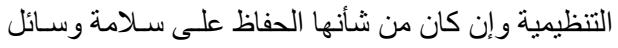

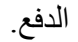
شفافية المعاملات:حيث ان عمليـات البيع و الثـر اء كلها
أو سلسلة الكتل وهى تقنية ظهرت عام (Blockchain)

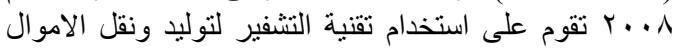

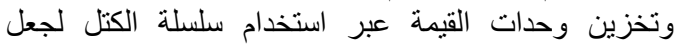
المعاملات آمنة. و البلوك تشين عباره عن عن سجل أو أو دفتر

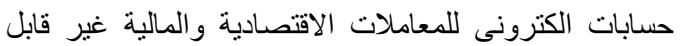
للتلاعب به. وبمعنى آخر أن أى تعامل اقتصادى أو الو مالى لى

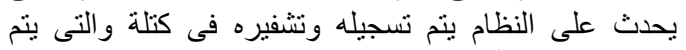

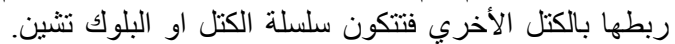

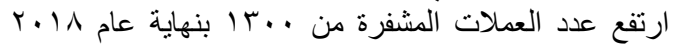

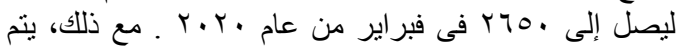

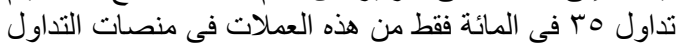

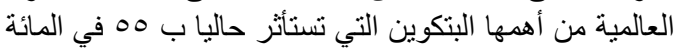
من القيمة السوقية للأصول المشفرة المتداولة الثينة عالميا، تليها

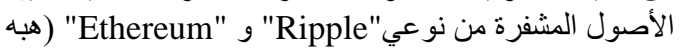

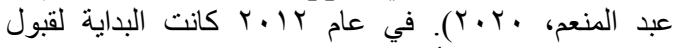

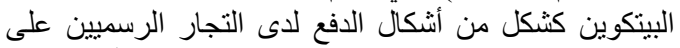

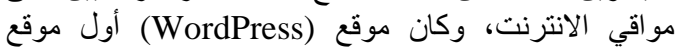

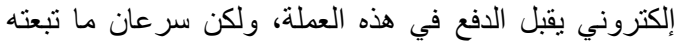

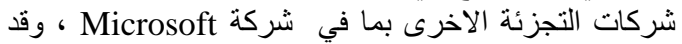
اعتبرت هذه الخطوة هي الاولى نحو قبولة الإني البيتكوين و العملة

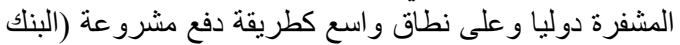

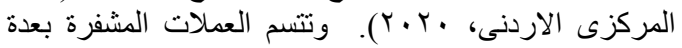
سمات منها (Nian and Chuen, 2015):

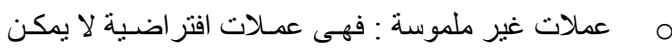

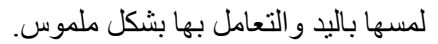

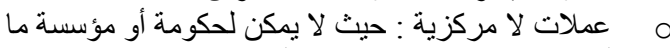

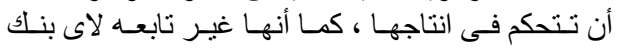

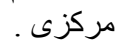

تعتمد بشـكل أسـاسي ووحيـ على التكنولوجيـا وخدمات

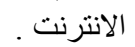

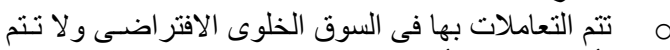

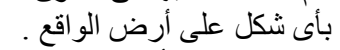

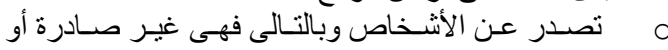
مضمونة من قبل البنوك المركزية الأنية فئية فئية

ومن أثهر العملات المشفرة الموجودة على الساحة العالمية :

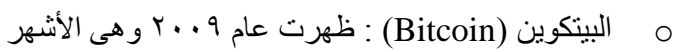
والاعلى سعر الئك على الإطلاق.

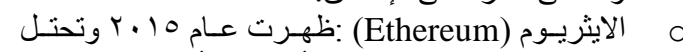

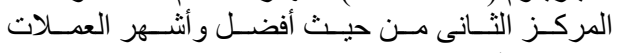
الافتر اضية . الركن

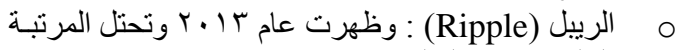
الثالثة فى هذا العالم الافتر اضى. : وظهرئ

وهناك عملات رقمية صادرة من البنك المركزي وهى العملة

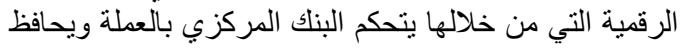

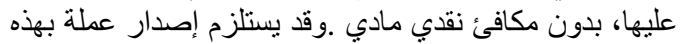


الأوراق المالية للعملة الرقمية للبنأ المركزي (CBDC) في

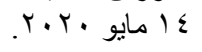

r بنزويلا

تعتبر فنزويلا أولى الدول التى لجأت الى الى استخدام العملات

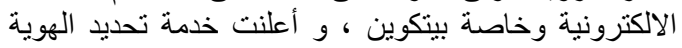

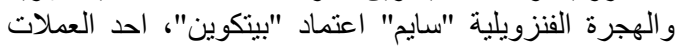

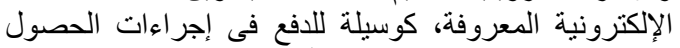

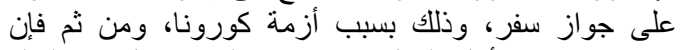

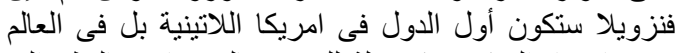

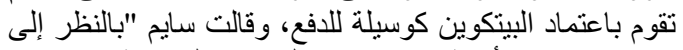

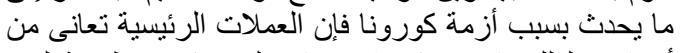

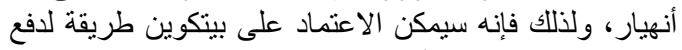

رسوم جواز سفر فنزويلى (Johnson, 2020).

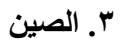

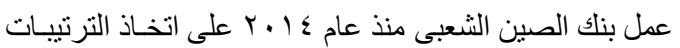

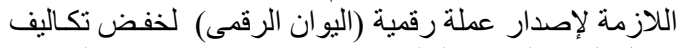

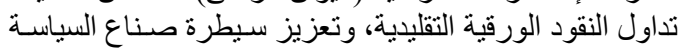

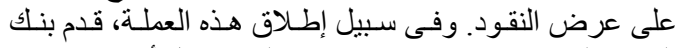

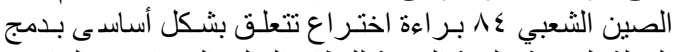

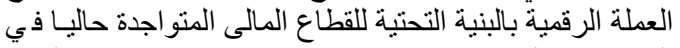

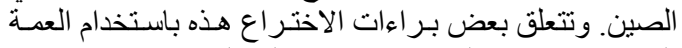

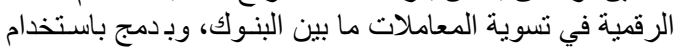

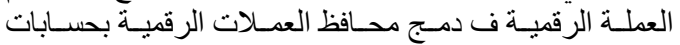

التجزئة الحالية Banking Retail Accounts.

\section{ع. الدول العربية}

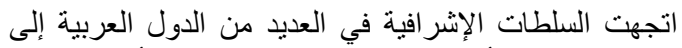

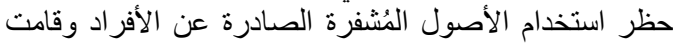

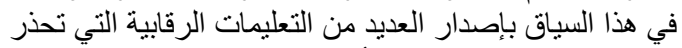

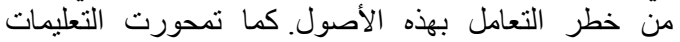

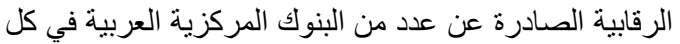

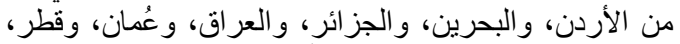

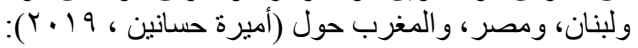

O مظر استخدام الأصول المُشفرة من قبل البنوك و الأفر اد.

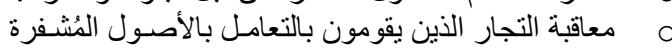

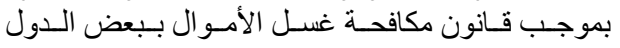

العربية.

التحذير من كون تعاملات الأصول المُشفرة تنطوي على مخاطر عالبة لتقلب الأسعار.

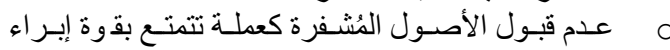

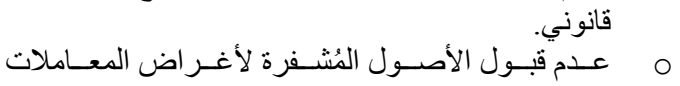
التجارية الرسمية.

جدير بالذكر انـه اهتمـت الدول العربيـة بدراسـة مدى جدوى ترسيه
معلنة ومعروفة لجميع المستخدمين.

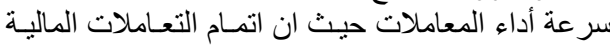

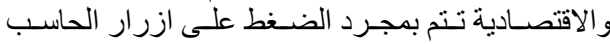

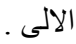

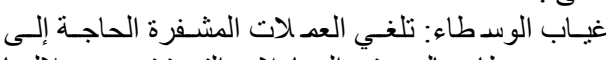

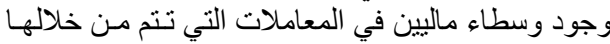

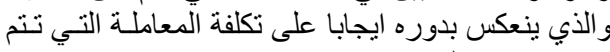

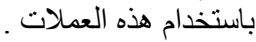

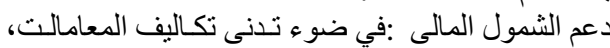

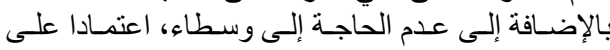

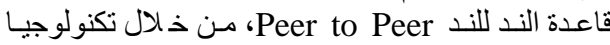

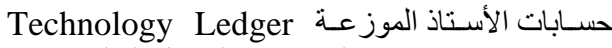

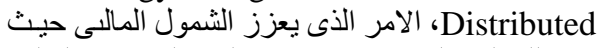

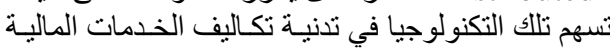
عوضا عن سر عة تنفيذ تلك الخدمات.

\section{ج. مخاطر التعامل بالعملات المشفرة}

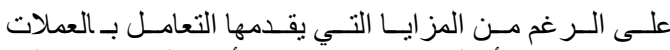

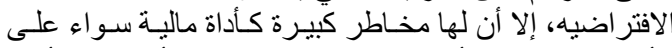

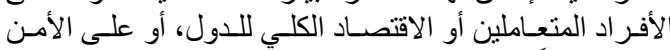
القومي أيضاً، وتكمن مخاطر ها في الأني:

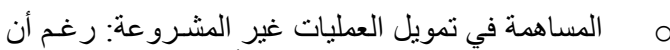

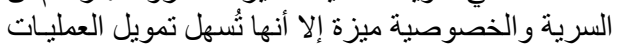

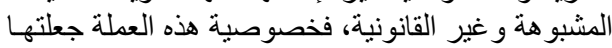

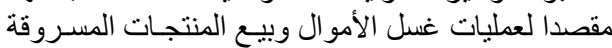
و الممنوعة، وتمويل الجماعات الإرهابية.

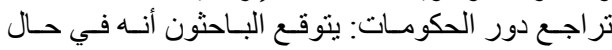

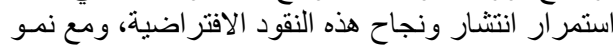

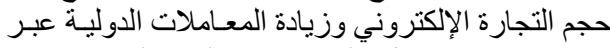

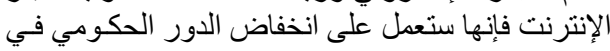

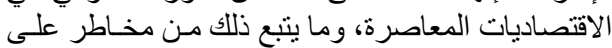

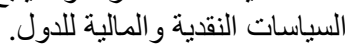

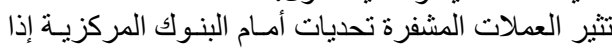

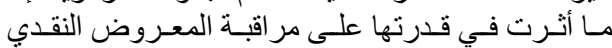
وبالتالي على تتفيذ السياسة النقدية.

\section{د. واقع التعاملات بالعملات المشفرة فى بعض الدول

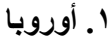

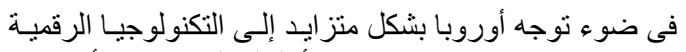

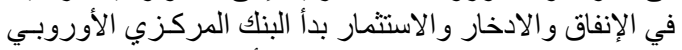

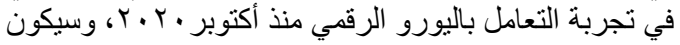

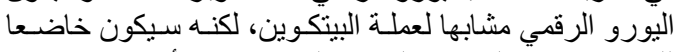

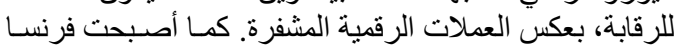

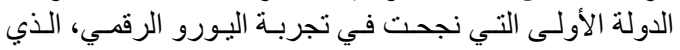

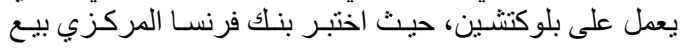




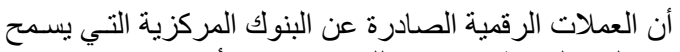

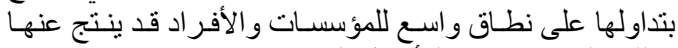

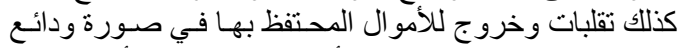

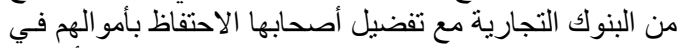

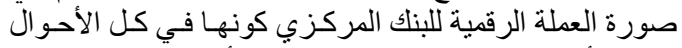

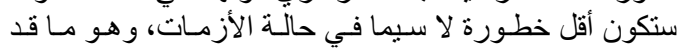

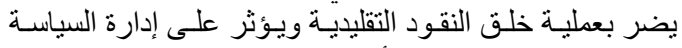

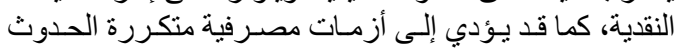

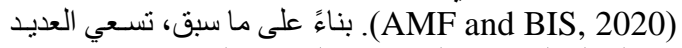

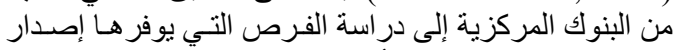

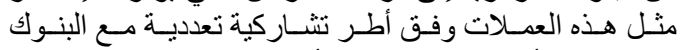

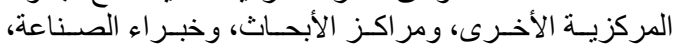

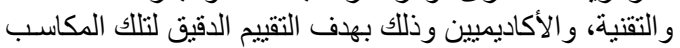

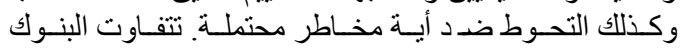

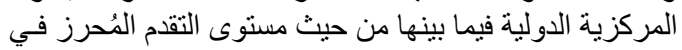

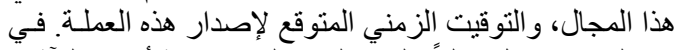

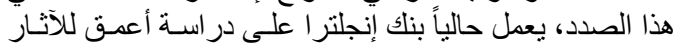

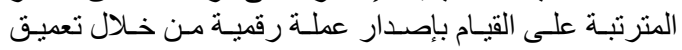

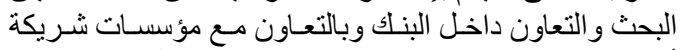

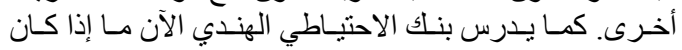

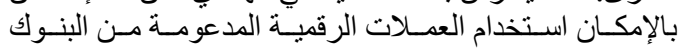
المركزية كعملات قانونية.

\section{هـ. التظظيمات والتشريعات الخاصة بالعملات المشفرة والمستقرة}

تبذل المؤسسات الدولية وواضعى المعايير العالمية جهودا

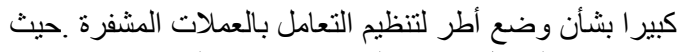

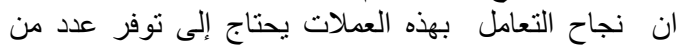

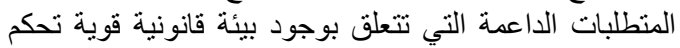

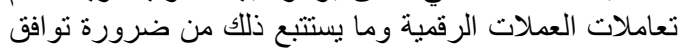

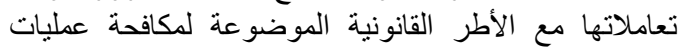

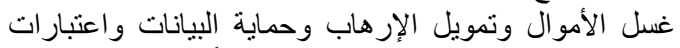

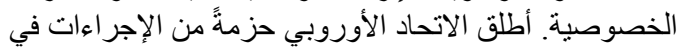

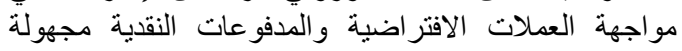

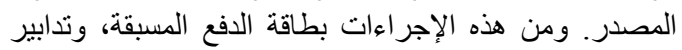

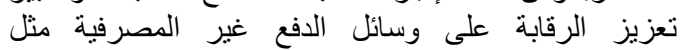

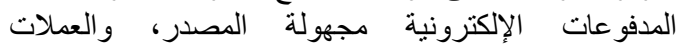

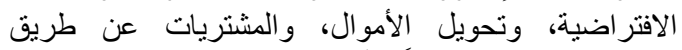

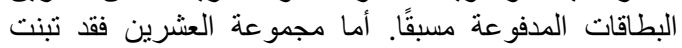

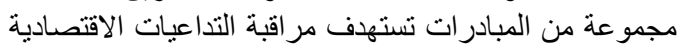

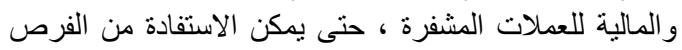

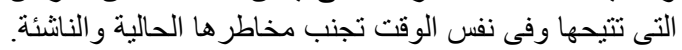

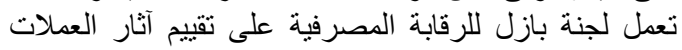

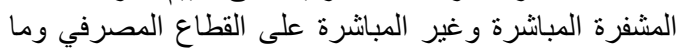
هى الإجر اءات الإحتر ازيه الو اجب إتخاذها حيال مخاطر ها.

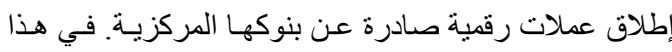

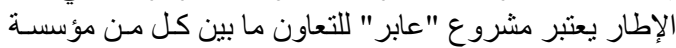

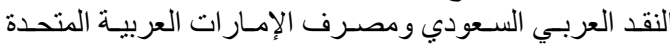

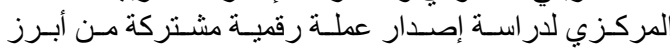

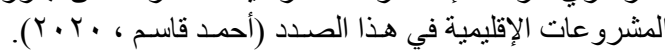

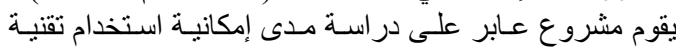

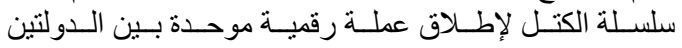

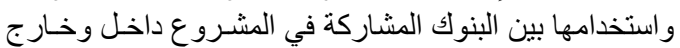

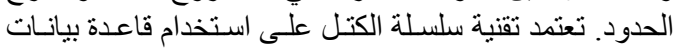

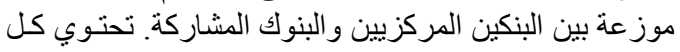

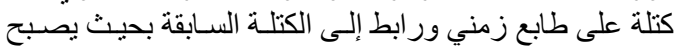

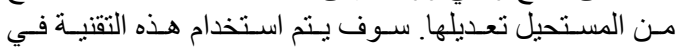

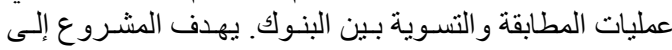

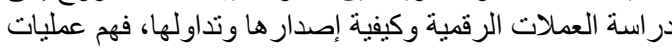

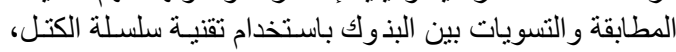

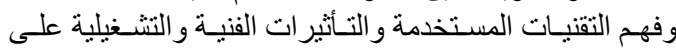

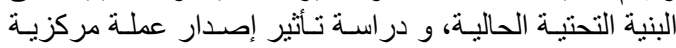

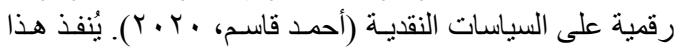

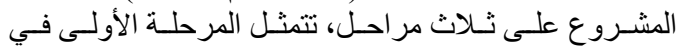

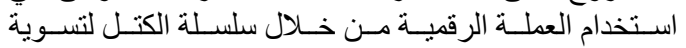

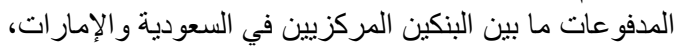

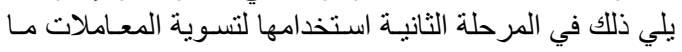

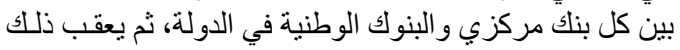

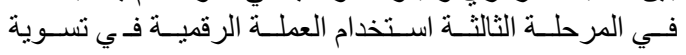

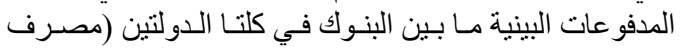

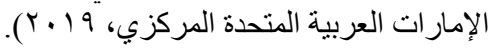

\section{هـ. دور البنوك المركزية فى التعامل مع هذه العملات}

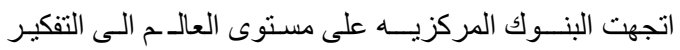

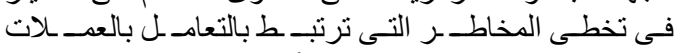

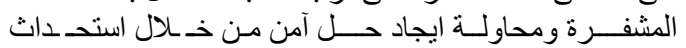

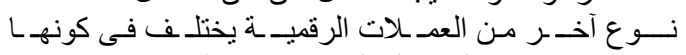

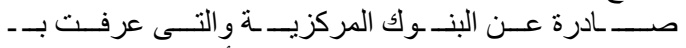
Central Bank Digital Currency يعرفها بنلك التسويات الدوليـة بكونهـا "شكل جديد من أنشكال

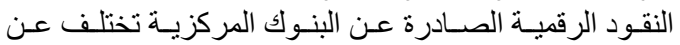

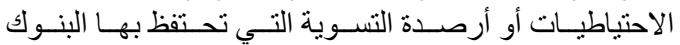

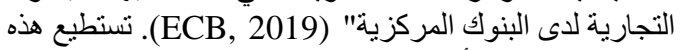

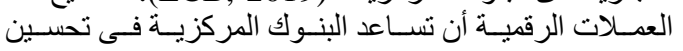

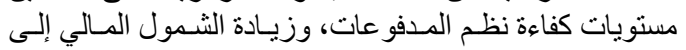

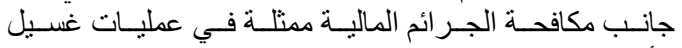

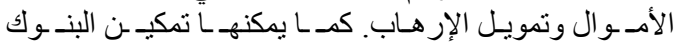

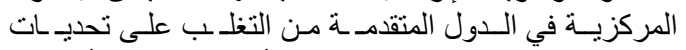

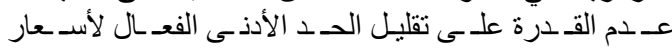

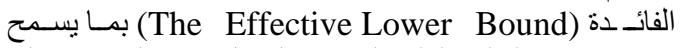
بتحفيز جانب الطلب الكلي. لكن في المقابل نشير الدراسات إلى 
كورونا المستجد سوف نتناول بيانات التداول بدءا من نوفمبر

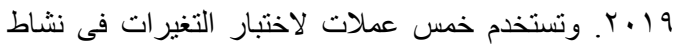

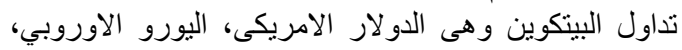

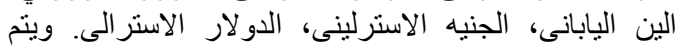

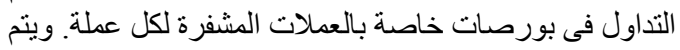

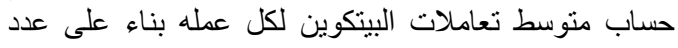

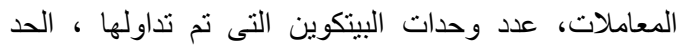

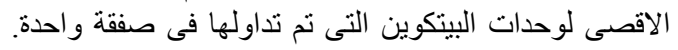

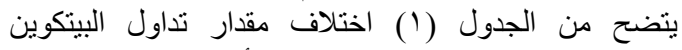

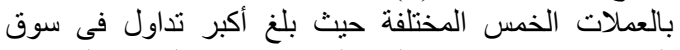

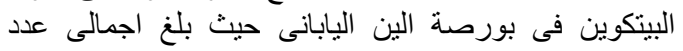

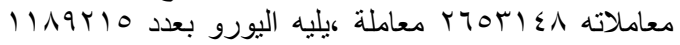

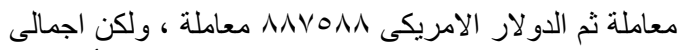

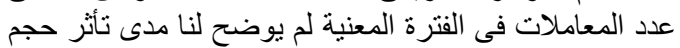

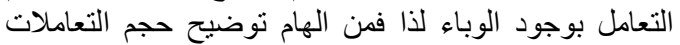

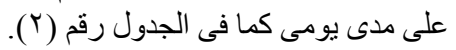
ثالثملات أثر فيروس كورونا المستجد على التعاملات

اختلفت وجهات النظر البحثية فيما يخص مدى تأثر التعاملات

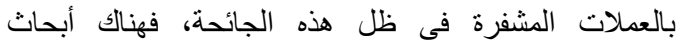

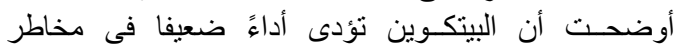
التحوط، كما انها لا تعد ملاذا آمنا فى هذا الوفي الوقت الذئى يتسم

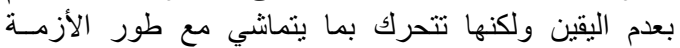
بمعنى انها ترياق مالي يحمي (Corbet et al., 2020)

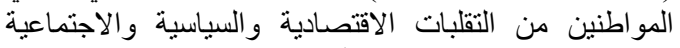

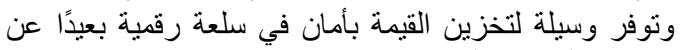

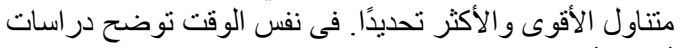

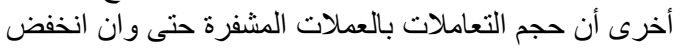

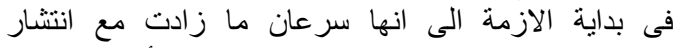

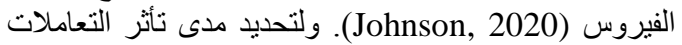
بالبيتكوين، باعتبار ها أعلى عملة مشفرة تداو لا، بسبب فيرو لنعاملات

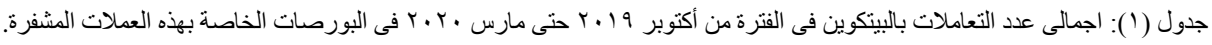

\begin{tabular}{|l|l|l|l|l|l|}
\hline Exchange & Currency & $\begin{array}{c}\text { Period 1 } \\
\text { Oct-Nov 2019 }\end{array}$ & $\begin{array}{c}\text { Period 2 } \\
\text { Dec 2019 }\end{array}$ & $\begin{array}{c}\text { Period 3 } \\
\text { Jan-Mar 2020 }\end{array}$ & $\begin{array}{c}\text { Total } \\
\text { Oct 2019-Mar 2020 }\end{array}$ \\
\hline Kraken & US\$ & 887,588 & 347,487 & $2,054,517$ & $3,289,592$ \\
\hline Kraken & $€$ & $1,189,215$ & 521,560 & $2,827,589$ & $4,538,364$ \\
\hline Coincheck & $¥$ & $2,635,148$ & $1,040,382$ & $6,136,089$ & $9,811,619$ \\
\hline Coinfloor & $£$ & 28,480 & 13,721 & 45,145 & 87,346 \\
\hline BTC Markets & A $\$$ & 51,076 & 19,378 & 108,961 & 179,415 \\
\hline
\end{tabular}

Source: (Johnson, 2020).

تتأثر بالظروف الر اهنة والناجمه عن فيروس كورونا المستجد

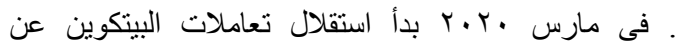

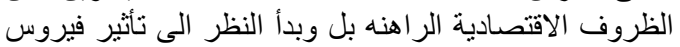

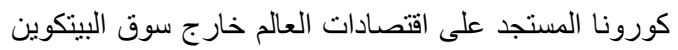

.(Szepietowski Virginia, 2020)
ويوضح جدول (Y) أن هناك انخفاض فى مستوى التعامل

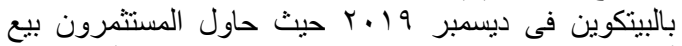

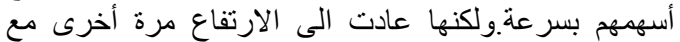

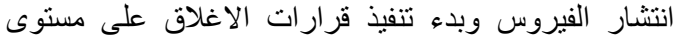

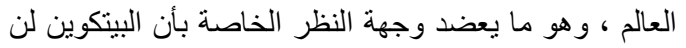

\begin{tabular}{|c|c|c|c|c|}
\hline Exchange & Currency & $\begin{array}{c}\text { Period } 1 \\
\text { Oct-Nov } 2019 \\
\end{array}$ & $\begin{array}{c}\text { Period } 2 \\
\text { Dec } 2019 \\
\end{array}$ & $\begin{array}{c}\text { Period 3 } \\
\text { Jan-Mar } 2020 \\
\end{array}$ \\
\hline Kraken & US\$ & 14,551 & 11,209 & 22,577 \\
\hline Kraken & $€$ & 19,495 & 16,825 & 31,072 \\
\hline Coincheck & $¥$ & 43,199 & 33,561 & 67,430 \\
\hline Coinfloor & $£$ & 467 & 443 & 496 \\
\hline BTC Markets & $\mathrm{A} \$$ & 837 & 625 & 1,197 \\
\hline
\end{tabular}

Source: (Johnson, 2020).

التحوط للعملات المشفرة في مواجهة حالة عدم اليقين التي

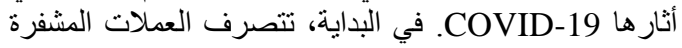

والوضع بالنسبة إلى Ethereum و Ripple منثابه مع وضع البيتكوين ولكن مع تفاعلات أضعف. هذا يبين دور 
مثل الأصول التقليدية ، لكنها تبدأ لتصبح تحوطًا مع تعمق توصيات البحث O تطوير الأطر الرقابية والقانونية لضمان تحديد هوية

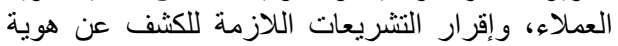
مستخدمي هذه العملات الافتر اضئة

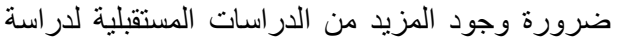

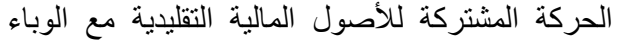

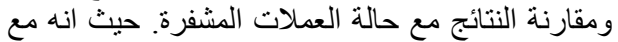
مرور الوقت، سيزداد عدد الملاحظات ويمكن أن يوفر المثر رؤى جديدة لسلوك العملات المشفرة في المراحل

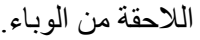

الاستفادة من الابتكار ات الحديثة في مجال الهوية الرقمية و العمليات الرقمية للنهوض بالثمولَ المالي.

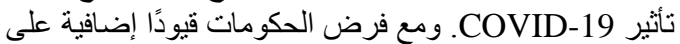

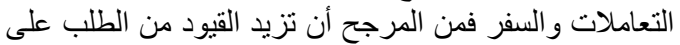

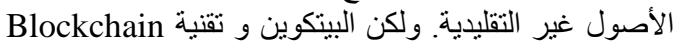

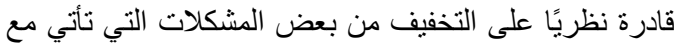

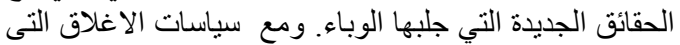

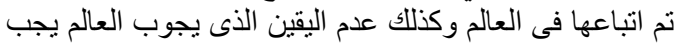

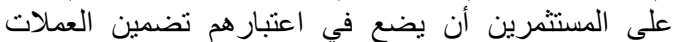

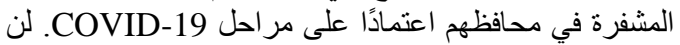

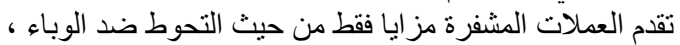
ولكن يمكن استخدامها أيضًا كأداة للادفع وتحويل النحوطن الأموال، .(Demir et al., 2020)

البنك المركزى الاردنى (· · • ·) ، العملات المشفرة ، ورقة

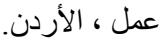

أميرة حسانين ، أسامة وجدى (19 1 (Y) ، خصائص العمالت

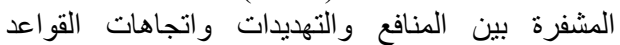
التنظيمية ، المجلة العلمية للاقتصاد و التجارة ، ، مجلد r^イ_r

مصرف الإمار ات العربية المتحدة المركزي (9 ( • ب) ، عابر:

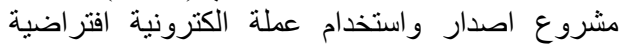

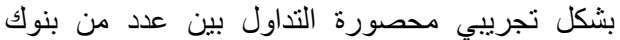

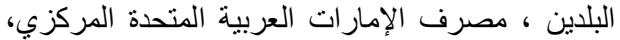

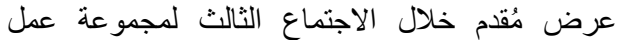

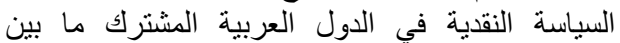
صندوق النقد العربي وبنك التسويات الدولية.

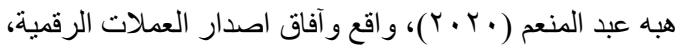
صندوق النقد العربي ، موجز سياسات ، العدد (1) (1).

Arab Monetary Fund (AMF) and Bank for International Settlements (BIS), (2020), Central bank papers on monetary policy frameworks in the Arab countries, Third working party meeting on monetary policy in the Arab region, Abu Dhabi, United Arab Emirates.

Bank for International Settlement (BIS) (2018),
تلعب العملات المشفرة دورا لا يمكن تغافله فى السنوات

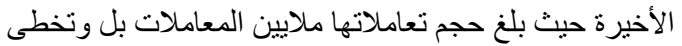

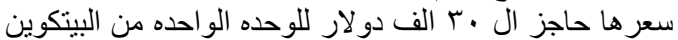

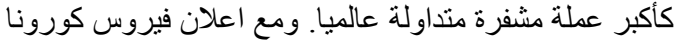

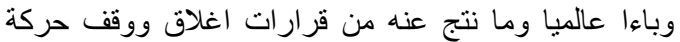

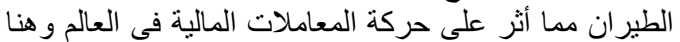

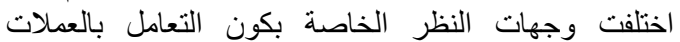

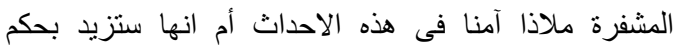

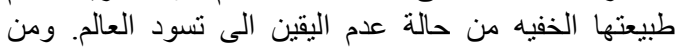

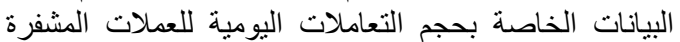

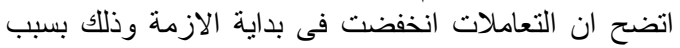

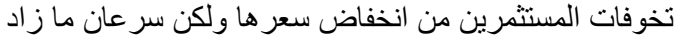
حجم التعاملات بها مرة أخرى مع بداية انتشار الفيرون الفيروس.

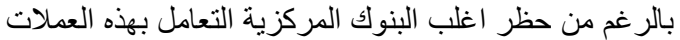

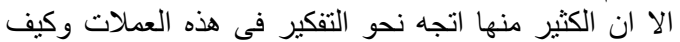
يمكن تقنين استخدامها لتصبح تحت طائلة السلطات النقات النقية،

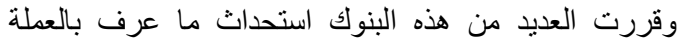
الرقمية كخطوة قانونية للتعاملات الحديثة.

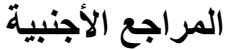

Central Bank Digital Currencies, Retrieved from: https://www.bis.org/cpmi/publ/d174.pdf.

Bank of Albania, (2017), Press Release on the Risk Associated with the Use of Virtual Currency, Albania. Retrieved from: https://www.bankofalbania.org/press/on_th e_risks_associated_with_the_use_of_virtua 1_cu rrency.html. 
Corbet, S., Larkin, C., Lucey, B. (2020), "The contagion effects of the COVID-19 pandemic: Evidence from gold and cryptocurrencies", Finance Research Letters, Vol. 35 No. 1, pp. 101554.

Demir, E., Bilgin Mehmet H., Karabulut, G. and Doker Asli C. (2020), The Relationship between Cryptocurrencies and COVID-19 Pandemic. Available at: http://dx.doi.org/10.2139/ssrn.3585147.

European Central Bank (ECB), (2019), Understanding the crypto-asset phenomenon, its risks and measurement issues, European Central Bank, Germany.

Johnson, J. (2019), "Bitcoin and Venezuela's Unofficial Exchange Rate". Ledger, Vol. 4. https://doi.org/10.5195/ledger.2019.170.

Johnson, J. (2020), The impact of COVID-19 on bitcoin trading activity: A preliminary assessment. Available at: http://dx.doi.org/10.2139/ssrn.3583162.
Krause, Makari (2016), Bitcoin: Implications for the Developing World, CMC Senior Theses, pp. 1261. Retrieved from: https://scholarship.claremont.edu/cmc_thes es/1261.

Nian, L. P. and Chuen D. L. K. (2015), Handbook of Digital Currency, Academic Press, pp. 5-30.

Stewart, K., Gunashekar, S. and Manville, C. (2017), Digital Currency, and the Future of Transacting, Retrieved from: https://www.rand.org/content/dam/rand/pu bs/perspectives/PE200/PE254/RAND_PE2 54z1.arabic.pdf.

Szepietowski, V. (2020), How will the COVID19 crisis affect the cryptocurrency market? Available

at: https://www.researchgate.net/publication/341255437_How_w ill_the_COVID-19_crisis_affect_the_cryptocurrency_market. 